WIS/25/05-Dec-DPP

hep-th/0512211

\title{
Solving Flavor Puzzles with Quiver Gauge Theories
}

\author{
Yaron E. Antebi, Yosef Nir and Tomer Volansky \\ Department of Particle Physics, Weizmann Institute of Science, Rehovot 76100, Israel
}

(Dated: August 22, 2021)

\begin{abstract}
We consider a large class of models where the $S U(5)$ gauge symmetry and a Froggatt-Nielsen $(\mathrm{FN})$ Abelian flavor symmetry arise from a $U(5) \times U(5)$ quiver gauge theory. An intriguing feature of these models is a relation between the gauge representation and the horizontal charge, leading to a restricted set of possible FN charges. Requiring that quark masses are hierarchical, the lepton flavor structure is uniquely determined. In particular, neutrino mass anarchy is predicted.
\end{abstract}

PACS numbers:

\section{INTRODUCTION}

The charged fermion flavor parameters - quark masses and mixing angles and charged lepton masses - exhibit a structure that is not explained within the Standard Model (SM). The two puzzling features - smallness and hierarchy - are very suggestive that an approximate horizontal symmetry is at work. The simplest framework that employs such a mechanism to explain the flavor puzzle is that of the Froggatt-Nielsen (FN) mechanism 1]. The various generations carry different charges under an Abelian symmetry. The symmetry is spontaneously broken, and the breaking is communicated to the SM fermions via heavy fermions in vector-like representations. The ratio between the scale of spontaneous symmetry breaking and the mass scale of the vector-like fermions provides a small symmetry-breaking parameter. Yukawa couplings that break the FN symmetry are suppressed by powers of the breaking parameter, depending on their FN charge.

Model building within the FN framework usually proceeds as follows. One chooses a value for the small symmetry-breaking parameter(s), and a set of FN charges for the fermion and Higgs fields. These choices determine the parametric suppression of masses and mixing angles. One then checks that the experimental data can be fitted with a reasonable choice of order-one coefficients for the various Yukawa couplings. Thus all FN predictions are subject to inherent limitations:

- The FN charges are not dictated by the theory.

- The value of the small parameter is not predicted.

- There is no information on the $\mathcal{O}(1)$ coefficients.

The predictive power of the FN framework is thus limited. There is one relation among the quark flavor parameters that is independent of the choice of horizontal charges [2], and there are three in the lepton sector [3].
The resulting predictions, that suffer from order one uncertainties, are consistent with the data. Additional relations apply in the supersymmetric extension of the SM, but to provide new tests of the FN mechasnim, supersymmetric contributions to flavor changing processes must be explored, and the universal effects of RGE running should be small [4]. The predictive power is sharply enhanced in the framework of GUT. With an $S U(5)$ gauge symmetry, the number of independent fermion charges is reduced from fifteen to six.

To make further progress, one would like to embed the FN mechanism in a framework where some or all of the inherent limitations described above are lifted. This may happen in string theory. While realistic constructions of the supersymmetric SM in string theory are still under study [5, 6, 7, 8, 9, 10, 11, 12, 13, 14], much progress has been made in the search for string-inspired phenomenologically viable extensions of the SM such as the FN framework. The basic idea is that the FN symmetry is a pseudo-anomalous $U(1)$ symmetry [15, 16, 17, 18, 19]. Then the small parameter depends on the FN charges and, furthermore, if one assumes gauge coupling unification, there is a single constraint on the $\mathrm{FN}$ charges that can be translated into a relation between the fermion masses and the $\mu$-term. This idea is based on ingredients of the heterotic string and has led to a detailed investigation of the resulting phenomenology (see, for example, refs. 20, 21, 22, 23].)

On the other hand, we are not aware of any attempt to-date to construct FN models which arise from D-brane configurations 24]. In this paper, we take a step in this direction and consider FN models from quiver gauge theories. These theories arise at low energy as the effective theories on D-branes placed at singular geometries (see 25, 26, 27, 28, 29, 30] and references therein). As opposed to the heterotic case, these theories typically have numerous anomalous $U(1)$ 's. The anomalies are cancelled through the generalized Green-Schwartz (GS) mechanism [31, 32, 33]. We employ these anomalous $U(1)$ 's as flavor symmetries and construct FN models. 


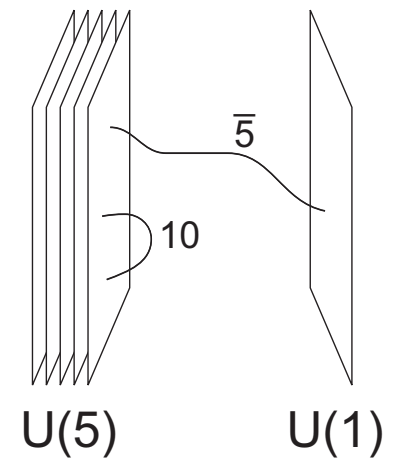

FIG. 1: A D-brane construction with an $S U(5)$ gauge group and a distinct $U(1)_{\mathrm{FN}}$. The fundamental fields are strings stretching between the two stacks and are thus charged under the FN group, while the antisymmetric fields connect only to the $U(5)$ stack and have no $U(1)_{\mathrm{FN}}$ charge.

As we show below, the structure of these theories tightly constrains model building and hence the realization of the FN mechanism. As a consequence, we will see that much can be said about the lepton sector. In particular, within the framework of $S U(5)$ GUT model with a single FN-symmetry breaking field, there is essentialy a single viable model which predicts mass anarchy in the neutrino sector.

It is worth noting that within the $S U(5)$ GUT model, it is a priori difficult to get the correct flavor hierarchy altogether. The reason for this is that $\mathbf{1 0}$ fields arise from open strings with both ends residing on the same set of $U(5)$ stack of branes, while $\overline{\mathbf{5}}$ fields come from strings with one end on the $U(5)$ stack and the other on a different brane which may provide the necessary $U(1)_{\mathrm{FN}}$ symmetry. This situation is depicted in Fig. 1] In particular this means that the $\mathbf{1 0}$ fields are not charged under the FN symmetry and there is no hierarchy in the up sector. As we show below, one can overcome this problem by extending the gauge symmetry, and the obtained structure is sufficiently restrictive to provide prediction regarding the neutrino sector.

The paper is organized as follows. In section [I] we discuss quiver gauge theories and their orientifold generalizations. We further discuss Higgsing and the role of anomalous $U(1)$ 's (and the problems that accompany them) in such theories. In section [II we investigate the embedding of the FN mechanism within quiver gauge theories. We argue that it is difficult to construct models with non-renormalizable superpotential terms. We focus on the case of a single $U(1)_{\mathrm{FN}}$ symmetry that arises from anomalous $U(1)$ 's. We show that there are severe restrictions on the possible FN charges, which lead to a generic constraint on the maximal hierarchy in this framework. In section IV we construct FN models in $S U(5)$-GUT theories with a single FN field. We show that there are only three possible FN charges for the 10-plets, and two for the $\overline{\mathbf{5}}$-plets. This situation makes the theory highly predictive. In particular, requiring that quark masses are hierarchical, a single set of charges is singled out, leading to a unique flavor structure. The flavor structure of the lepton sector is fixed, and neutrino mass anarchy is predicted. We conclude in section $\nabla$ A full, consistent, quiver realization for the SU(5) GUT theory, with emphasis on anomaly cancellation, is presented in Appendix A We collect the six Tables of our paper at the very end. Tables I-III show possible FN charges and suppression factors in generic quiver theories, while tables IV-VI give all possible models within our $S U(5) \times S U(5)$ framework.

\section{QUIVER GAUGE THEORIES}

A wide class of $\mathcal{N}=1$ supersymmetric vacua is obtained by placing D-branes on singular manifolds of type II strings such as orbifolds and orientifolds 25, 26, 27, 29, 34, 35, 36, 37, 38, 39, 40, 41]. Placing D-branes at such singularities produces at low energy conformal gauge theories [27], while adding fractional D-branes breaks the conformal symmetry, rendering a four dimensional chiral gauge theory.

A quiver diagram is an efficient way for describing the gauge theory obtained from the open string sector (for a review, see [30]). The degrees of freedom of oriented strings can be described as strings starting and ending on D-branes. Consequently, the fields in the theory transform in the fundamental of a $U\left(N_{i}\right)$ factor of the gauge group and in the antifundamental of another $U\left(N_{j}\right)$ factor. It is therefore possible to describe the field theory by a quiver diagram, where we denote each $U\left(N_{i}\right)$ factor by a node in the graph and the fields are represented by directed lines connecting two such nodes. The orientation of the line represents the orientation of the string: a line coming out of a node corresponding to a $U\left(N_{i}\right)$ gauge group factor stands for a field in the fundamental $\mathbf{N}_{\mathbf{i}}$, while a line going into a node corresponding to $U\left(N_{j}\right)$ represents a field transforming in the antifundamental $\overline{\mathbf{N}_{\mathbf{j}}}$. A line originating and ending on the same node, describes a field in the adjoint representation of the corresponding $U(N)$ factor.

Gauge invariant field combinations, which may be present in the superpotential, can also be seen using the diagrammatic description. A field transforming in the fundamental of a given $U(N)$, must interact with a field in the antifundamental of the same $U(N)$ in order to get an invariant interaction. In other words, if there is a field coming into a given vertex, we must also have a field going out of that same vertex. This has to be the case for all the vertices, so an invariant interaction is described by a closed loop in the quiver diagram. In particular, a renormalizable cubic term in the superpotential is represented in the quiver by a closed triangle. In general, however, not every closed loop in the quiver which originates from a certain geometry appears in the superpotential. The general problem of extracting the spectrum and superpotential from a given geometry is still not solved, and 
only specific examples are known.

\section{A. Orientifold Quivers}

Strictly speaking, gauge theories arising from unoriented string theory are not quivers since the low energy field theory cannot be described by a directed graph. Nevertheless, these theories may also be described diagrammatically by 'extended' quivers, where the lines representing the strings are no longer directed. Instead, the two ends of each string can independently be in either the fundamental or the antifundamental. Thus each line must be drawn with an arrow at each of the two ends, indicating what is the representation of the corresponding string under each of the two gauge group factors. Unoriented strings with both ends coming out of the same set of branes may reside in either the symmetric or the antisymmetric combination of $\mathbf{N} \times \mathbf{N}$. Which of these two options is realized is directly related to the orientifold projection in the original theory: it is the antisymmetric (symmetric) part for the $S O(N)(S p(N))$ orientifold projection. Having said that, we stress that the effective field theories of unoriented strings on singular manifolds are only known for $\mathbb{Z}_{n}$ orbifolds. Nevertheless, it is expected that the unoriented nature of the string should lead to the same 'extended' quiver type diagrams in more general singularities.

Again, invariant interactions follow from the (extended) quiver. It corresponds to a set of lines for which each node has the number of ingoing fields equal to the number of outgoing fields.

\section{B. Higgsing}

Higgsing, that is the spontaneous breaking of the gauge group via vacuum expectation values (VEVs) of scalar fields, changes in general the low energy description of a given theory. In particular, by Higgsing a quiver gauge theory, one obtains a (not necessarily supersymmetric) new quiver gauge theory where the low energy degrees of freedom are bi-fundamentals of the conserved gauge groups. We can thus write an effective quiver by identifying or splitting the vertices and lines so that each new node represents an unbroken $U(N)$ gauge group.

As an example, consider string theory on a $\mathbb{Z}_{3}$ orbifold of the conifold (also known as $Y^{3,0}$ ), with $N$ branes at each representation of $\mathbb{Z}_{3}$. The resulting $[U(N)]^{6}$ gauge theory is described by the quiver of Fig. 22. If we Higgs the theory by giving $Z^{1}$ a VEV proportional to the unit matrix,

$$
Z^{1}=\left(\begin{array}{cccc}
a & & & \\
& a & & \\
& & a & \\
& & & \ddots
\end{array}\right),
$$

the corresponding $U(N) \times U(N)$ group is broken to the diagonal $U(N)$, and the $[U(N)]^{5}$ gauge theory of Fig. [2] is obtained. The $Z^{1}$ field itself is eaten up by the longitudinal modes of the broken gauge fields. Higgsing $Z^{2}$ and $Z^{3}$ in the same way, one finds the $[U(N)]^{3}$ theory described by Fig. 2r, which is exactly the quiver of the orbifold $\mathbb{C}^{3} / \mathbb{Z}_{3}$ [37].

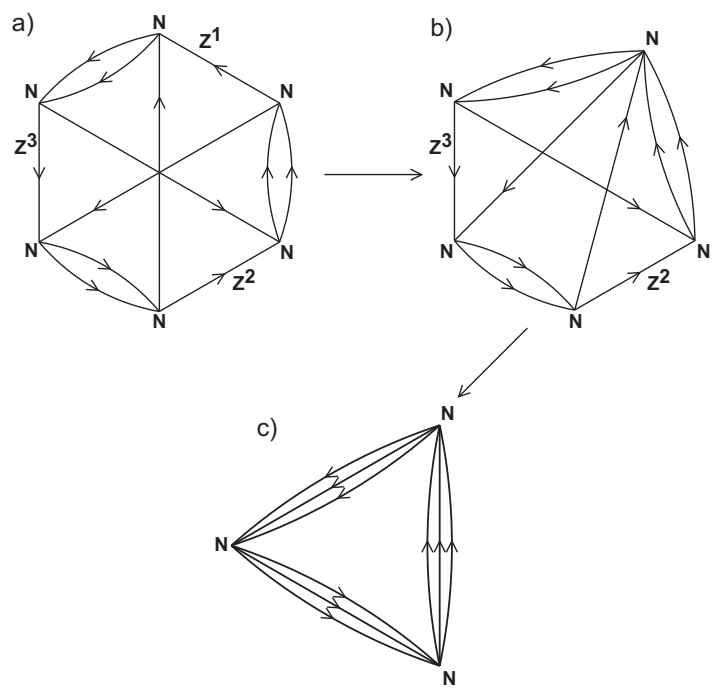

FIG. 2: Successive Higgsing of the $Y^{3,0}$ quiver diagram to a $\mathbb{C}^{3}$ quiver: a) The $Y^{3,0}$ quiver diagram; b) The effective quiver diagram induced by Higgsing $Z^{1} \propto \mathbf{1}$; c) The $\mathbb{C}^{3} / \mathbb{Z}_{3}$ quiver induced by Higgsing also $Z^{2}, Z^{3} \propto \mathbf{1}$.

If, instead, we break the $Y^{3,0}$ quiver by choosing a different form of the VEV for $Z^{1}$,

$$
Z^{1}=\left(\begin{array}{llll}
a & & & \\
& \ddots & & \\
& & b & \\
& & & \ddots
\end{array}\right),
$$

the symmetry breaking is $U(N) \times U(N) \rightarrow U(n) \times U(N-$ $n)$. This breaking is no longer supersymmetric and $Z^{1}$ in not eaten up completely. The lines and the nodes split and the quiver takes a very different form, as can be seen in Fig. 3. Other breaking patterns of $U(N) \times U(M)$ symmetries with bi-fundamentals can be obtained. The most general breaking is

$$
U(N) \times U(M) \rightarrow U\left(N-M+n_{0}\right) \times \prod_{i=0}^{k} U\left(n_{i}\right)
$$

with $\sum_{i=0}^{k} n_{i}=M$. It leads to more complicated quiver diagrams which, in general, will not be supersymmetric due to a non-vanishing D-term. However, by giving VEVs to a pair of fields in a vector representation, supersymmetry may easily be preserved.

Different patterns of breaking are generated when the Higgsed field is in the adjoint representation or, for 'extended' quiver, in the fundamentals of both gauge groups or in the symmetric (or anti-symmetric) representation. 


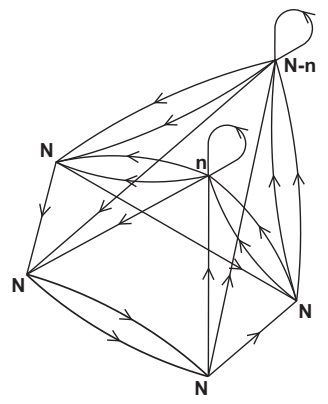

FIG. 3: Breaking the $Y^{3,0}$ quiver by a diagonal VEV with two different eigenvalues with multiplicities $n$ and $N-n$.

In all cases, the resulting theories can be described by a new quiver diagram.

\section{Anomalous $U(1)$ 's}

Typically, many of the $U(1)$ factors associated with the $U(N)$ gauge groups are anomalous, with anomalies cancelled by the generalized Green-Schwartz (GS) mechanism [25, 31, 32, 33]. In contrast to the case of the heterotic string, in type II string theory there can be several such anomalous $U(1)$ factors. Furthermore, the corresponding gauge fields are massive independent of whether the symmetry is spontaneously broken (the spontaneous breaking is induced by non-vanishing Fayet-Illiopolous (FI) terms) [2, 43]. In the case that the symmetry is not broken by scalar VEVs, these $U(1)$ factors remain as global symmetries 44] and the corresponding $S U(N)$ factors remain good gauge symmetries. Thus each node in the quiver has a corresponding (local) global (non)anomalous $U(1)$.

Phenomenologically, these $U(1)$ factors pose considerable problems to model building, essentially since the global $U(1)$ symmetries of the SM or its supersymmetric extensions (SSM) are not directly related to the local gauge groups. Consider, as an example, a supersymmetric quiver theory which contains the $S U(2)_{W}$ gauge group [5]. Under the corresponding $U(1)$, all the doublets $\left(Q_{i}\right.$, $L_{i}, H_{u}$ and $\left.H_{d}\right)$ are charged \pm 1 . The superpotential of the SSM has the following form:

$$
W=Y_{i j}^{d} H_{d} Q_{i} d_{j}+Y_{i j}^{u} H_{u} Q_{i} u_{j}+Y_{i j}^{L} H_{d} L_{i} e_{j}+\mu H_{u} H_{d} .
$$

It is clear that, to allow for $Y^{u} \neq 0$ and $Y^{d} \neq 0$, the $U(1)$ charges of $H_{u}$ and $H_{d}$ must be the same. But then the $\mu$ term is forbidden. A similar problem exists in the $S U(5)$ GUT models [8, 9] and their extensions, as we discuss in section IV

There are three possible solutions to the above problem:

1. The particle content of the low energy theory is extended in such a way that the symmetry is realized. For example, an extended higgs sector 5] enlarges the global symmetry.
2. The $U(1)$ is broken spontaneously. The only way to do this without breaking the associated $S U(N)$ gauge group is by letting a singlet composed of $N$ fundamentals to obtain a VEV. This breaks the $U(1)$ down to a $\mathbb{Z}_{N}$. Such composite singlets may exist if they are charged under a different, confining gauge group.

3. The anomalous $U(1)$ is broken by non perturbative effects. Depending on the matter content, such effects break the symmetry down to $\mathbb{Z}_{k N}$ for some integer $k$.

While difficult to analyze, these solutions allow realistic extensions of the SSM through quivers. We discuss these possibilities further in section IV

\section{THE FN MECHANISM FROM QUIVERS}

\section{A. Renormalizable or Non-Renormalizable}

The Froggatt-Nielsen (FN) mechanism 1] provides an explanation to the flavor puzzle using a horizontal symmetry. Quarks and leptons of various generations are charged differently under a symmetry $\mathcal{H}$ which is spontaneously broken. The simplest realization is through the use of a single Abelian $U(1)$ which is broken by the VEV of a scalar field $S$. To allow for $\mathcal{H}$-invariant interaction terms involving the SM Higgs and fermion fields, powers of $S$ must of be involved, depending on the $\mathcal{H}$-charge of the Yukawa interaction. Thus, non-renormalizable interaction terms arise, suppressed by inverse powers of $M_{V}$, the scale at which the breaking of $\mathcal{H}$ is communicated to the SM. The effective Yukawa interactions are then suppressed by powers of $\langle S\rangle / M_{V}<1$, and are characterized by smallness and hierarchy, as required phenomenologically.

One way of embedding the FN mechanism in string theory would be by identifying the scale $M_{V}$ with the string scale, and obtaining a superpotential that has most of its terms non-renormalizable (the top, and perhaps other third generation Yukawa couplings, being the exception). This goal is difficult to achieve. Quiver gauge theories which arise from D-branes at singularities have a superpotential determined fully by the geometry. Geometrically engineering the required superpotential is hard. Most of the geometries which are under control arise from orbifold singularities 25] or toric geometries [37, 38, 40]. (These two classes are, of course, not separate: Abelian orbifolds are toric.) In the first type of geometry, the superpotential is obtained by truncating $\mathcal{N}=4$ superconformal Yang-Mills which has cubic interactions. For the second type, in the case of $Y^{p, q}$ geometries, one obtains also quartic interactions. In any case, it is difficult to construct a quiver theory with a superpotential that has most of its terms non-renormalizable.

A second approach is to construct a renormalizable model above the scale $M_{V}$ which, at low energy, produces 

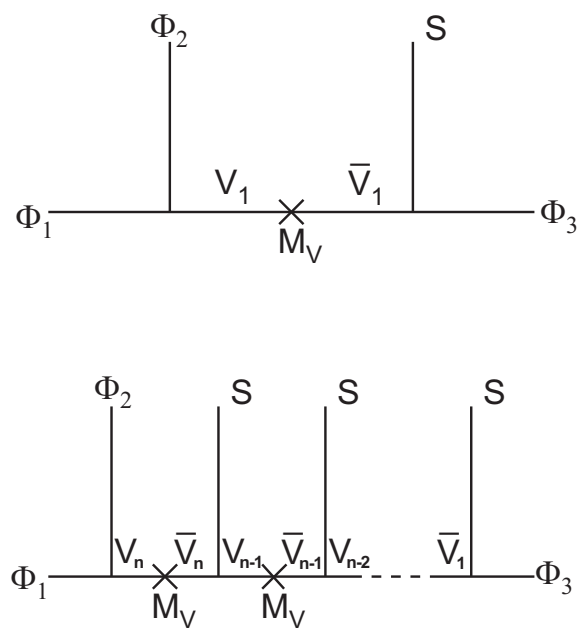

FIG. 4: Diagrams for generating interactions suppressed by different factors $\langle S\rangle / M_{V}$ using only cubic interactions.

the required interactions. Further motivation for such a construction comes from the possible identification of the FN symmetry with an anomalous $U(1)$, as discussed below.

A renormalizable model is easy to construct with the introduction of additional vector-like massive fields. As an example, consider a non-renormalizable superpotential term of the form

$$
W=\left(\frac{S}{M_{V}}\right)^{n} \Phi_{1} \Phi_{2} \Phi_{3} .
$$

To generate such interaction, we introduce additional vector-like massive fields $V_{k}, \bar{V}_{k}(k=1, . ., n)$, with masses at the scale $M_{V}$ and the following charges under the horizontal symmetry:

$$
\begin{gathered}
\mathcal{H}\left(\Phi_{1}\right)=n, \mathcal{H}\left(\Phi_{2}\right)=0, \mathcal{H}\left(\Phi_{3}\right)=0, \\
\mathcal{H}(S)=-1, \mathcal{H}\left(V_{k}\right)=-\mathcal{H}\left(\bar{V}_{k}\right)=-k
\end{gathered}
$$

Taking the renormalizable superpotential to be

$$
W=\Phi_{1} \Phi_{2} V_{n}+\bar{V}_{n} V_{n-1} S+\ldots+\bar{V}_{1} S \Phi_{3}+\sum_{i} M_{V} \bar{V}_{i} V_{i}
$$

and integrating out $V_{i}$ and $\bar{V}_{i}$, one finds the required interaction, eq. (5). Fig. 4 shows the relevant diagrams which generate this low energy interaction for $n=1$ and for general $n$.

\section{B. Identifying the FN Symmetry}

To relate the FN mechanism to a quiver field theory, one needs to identify a global symmetry that can play the role of a viable horizontal symmetry. We limit our search of viable models to theories with the following features:
- The FN symmetry arises from anomalous gauged $U(1)$ 's in the quiver. (In particular, we do not consider global symmetries of the open string sector that are related to isometries in the dual gravitational theory.)

- The spontaneous breaking of the symmetry comes from a VEV of a single field, $S$.

- The ratio of the two relevant energy scales is small,

$$
\epsilon \equiv\langle S\rangle / M_{V} \ll 1
$$

allowing for smallness and hierarchy in the effective Yukawa couplings.

We note several points that hold generically in a $\mathrm{FN}$ mechanism that arises from a quiver theory using an anomalous $U(1)$ :

1. For $S$ to be charged under an anomalous $U(1)$, it must either be stretched between two distinct vertices, or have its two ends on the same vertex but with both ends sitting in the same representation (either fundamental or antifundamental). The second situation is possible only in the orientifold case. Since $S$ is generically charged under the full $U(N)[\times U(M)]$, and not just under the anomalous $U(1)$ factors, it necessarily breaks some of the gauge groups. This means that the FN mechanism requires an extended group.

2. The charge of a given field under the FN symmetry is fixed by its representation under the corresponding non-Abelian gauge groups. For example, assume $S$ resides in the $\left(\mathbf{N}_{\mathbf{L}}, \overline{\mathbf{N}}_{\mathbf{R}}\right)$ representation of a $U\left(N_{\mathrm{L}}\right) \times U\left(N_{\mathrm{R}}\right)$ gauge group. It is neutral under the sum of the two $U(1)$ factors, $U(1)_{\mathrm{L}+\mathrm{R}}$, but has a charge +2 (in a specific normalization) under the difference, $U(1)_{\mathrm{L}-\mathrm{R}}$. Thus we must identify $U(1)_{\mathrm{FN}}$ with $U(1)_{\mathrm{L}-\mathrm{R}}$. Any field in the $\left(\mathbf{N}_{\mathbf{L}}, \mathbf{1}\right)$ representation has then a $\mathrm{FN}$ charge of +1 , while a field in the $\left(\overline{\mathbf{N}}_{\mathbf{L}}, \overline{\mathbf{N}}_{\mathbf{R}}\right)$ representation is neutral under the FN symmetry.

3. There may be other fields in the theory obtaining VEVs that break additional gauge groups or $U(1)$ 's. Our assumptions above mean that those VEVs are of the order of $M_{V}$ and do not contribute to the hierarchy. To distinguish between these fields and $S$, one may write the effective quiver after the Higgsing of all fields except $S$.

\section{FN charges}

As explained above, the charges of the various fields under the FN symmetry are very restricted. This, in turn, affects the possible hierarchical structure within the 


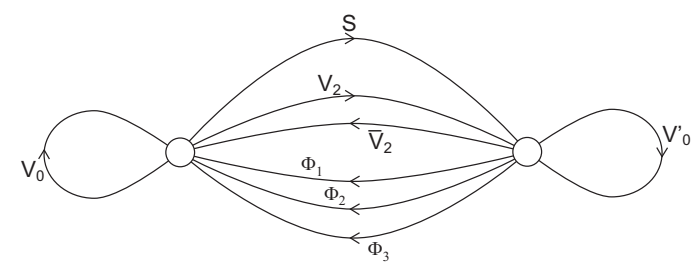

FIG. 5: A directed quiver diagram for an $\epsilon^{3}$-suppression of the effective $\Phi_{1} \Phi_{2} \Phi_{3}$ term in the superpotential.
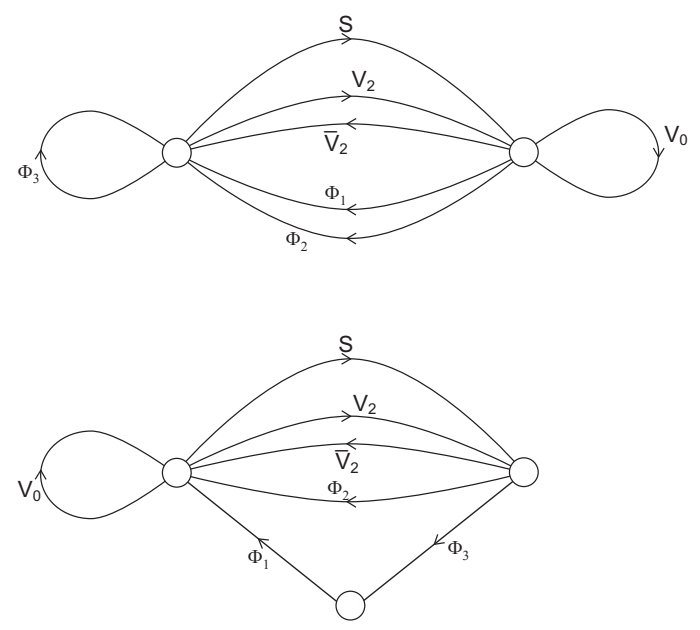

FIG. 6: Directed quiver diagrams for an $\epsilon^{2}$-suppression of the effective $\Phi_{1} \Phi_{2} \Phi_{3}$ term in the superpotential.

quiver theory. In fact, the strongest suppression possible is by $\epsilon^{3}\left(\epsilon \equiv\langle S\rangle / M_{V}\right)$ and even this suppression is unlikely to actually appear, as we explain below.

Consider first oriented strings. As explained above, for directed quivers, $S$ must be stretched between two distinct branes and so its charge is $(+1,-1)$ under the corresponding $U(1)_{\mathrm{L}} \times U(1)_{\mathrm{R}}$. The FN symmetry is then $U(1)_{\mathrm{FN}}=U(1)_{\mathrm{L}-\mathrm{R}}$ under which $S$ is charged +2 . Any other field can be charged with one of the following: $(0,0),( \pm 1,0),(0, \pm 1),( \pm 1, \mp 1)$. Thus the strongest suppression of an effective Yukawa term of the form $\Phi_{1} \Phi_{2} \Phi_{3}$ is obtained when all three fields are charged $(-1,+1)$, giving an $\epsilon^{3}$ suppression. The corresponding quiver diagram diagram is drawn in Fig. 5

While an $\epsilon^{3}$-supperssion can, in principle, be generated, it is unlikely to be relevant in practice, since it requires that all three fields $\Phi_{i}$ transform in the same way under the entire gauge group. In particular, there is no such case in $S U(5)$ GUT models, as we discuss further in section IV

To get an $\epsilon^{2}$-suppression, there are two possibilities for the charges: $(+1,-1),(+1,-1),(0,0)$ or $(+1,-1),(+1,0),(0,-1)$. Examples for the two sets of charges are drawn in Fig. [6

There are five sets of charges that yield an $\epsilon$ suppression. These (and the other sets of charges that yield suppression) are presented in Table @ The quiver
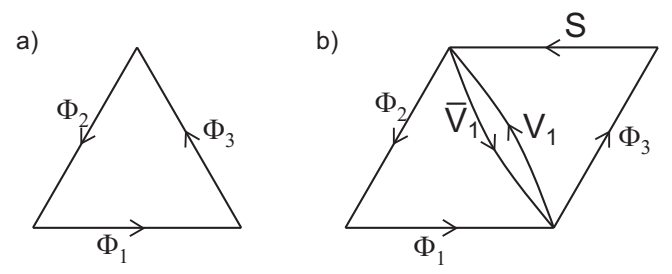

FIG. 7: Directed quiver diagrams which lead to (a) unsuppressed effective Yukawa coupling, and (b) $\epsilon$-suppression of the effective Yukawa coupling.

diagram that corresponds to the set $(0,0),(-1,0),(0,+1)$ is depicted in Fig. Z(b). Figure [7(a) shows the simplest triangle which produces a renormalizable (that is unsuppressed) Yukawa coupling.

For orientifolds, in addition to the above $U(1)_{\mathrm{L}} \times U(1)_{\mathrm{R}}$ charges, there could be fields with charges $( \pm 2,0),(0, \pm 2)$ and $( \pm 1, \pm 1)$. Furthermore, $S$ itself can have charges $(+1,-1)$ (similar to the oriented string), $(+1,+1)$ or $( \pm 2,0)$. The breaking patterns of the gauge groups will be different for these three choices. The $U(1)_{\mathrm{FN}}$ is then $U(1)_{\mathrm{L}-\mathrm{R}}, U(1)_{\mathrm{L}+\mathrm{R}}$ or $U(1)_{\mathrm{L}}$, respectively. For the first case, table II enumerates the possible suppression factors that arise in addition to the orbifold case of table [1. Note the additional configuration with an $\epsilon^{3}$ suppression, whose relevant quiver diagram is shown in Fig. 8 The second case, $U(1)_{\mathrm{L}+\mathrm{R}}$, can be easily obtained from the first one, $U(1)_{\mathrm{L}-\mathrm{R}}$, by multiplying the $U(1)_{\mathrm{R}}$ charges by minus one. Finally, the third case, $U(1)_{\mathrm{L}}$, again exhibits a configuration of $\epsilon^{3}$-suppression, as can be seen in table III] Here too, this configuration requires all three fields to be in the same representation of the non-Abelian gauge groups.

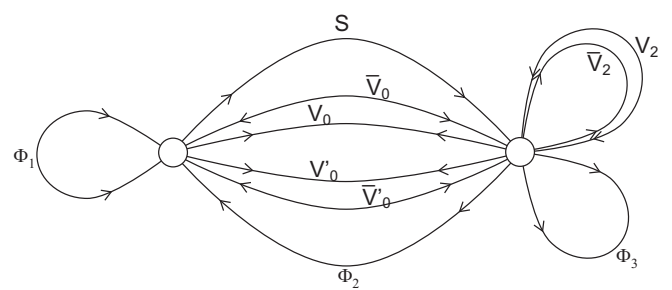

FIG. 8: A quiver diagram in the orientifold case that gives an $\epsilon^{3}$-suppression.

Our results in this section show the strong predictive power that is added to the generic Froggatt-Nielsen mechanism when embedded in string theory. In fact, the constraints are so strong - i.e. the strongest suppression of a Yukawa coupling is third order in a small parameter and, for practical purposes, probably only second order - that one may wonder whether our framework gives rise to any viable flavor model at all. Indeed, in the next section we show that to construct viable models, one has to invoke (rather plausible) non-perturbative effects. These effects relax some of the constraints that we presented in this section and, in particular, allow an $\epsilon^{4}$-suppression of 
the Yukawa couplings in the case of $S U(5)$ GUT models.

\section{IV. $S U(5)$ GUT MODELS AND NEUTRINO MASS ANARCHY}

In this section we consider $S U(5)$ GUT models with a FN flavor symmetry. We search for viable models that arise from quiver gauge theories. The theory turns out to have intriguing implications for the neutrino sector.

By an $S U(5)$ GUT model we mean that there is a range of energy scales where the gauge group is $S U(5)$, with matter fields that transform as $\mathbf{5}, \overline{\mathbf{5}}$, and $\mathbf{1 0}$. The presence of an antisymmetric multiplet of the gauge group requires that we consider orientifold theories and choose the appropriate projection. This projection is the one that results in an $S O(N)$ gauge group(s).

Our analysis focusses on the energy scale just above the GUT breaking scale. In general there can be many fields that break the various gauge groups that are present in the quiver theory. However, as discussed in the previous section, we consider a scale that is low enough so that the only field to play a relevant role in the breaking of the FN symmetry and possibly in breaking of a larger gauge group into $S U(5)$ is the FN field.

\section{A. General Considerations and Predictions}

The strongest mass hierarchy in the various fermion mass matrices appears in the up sector. Thus, a minimal requirement that we put on viable models is that they produce an up mass hierarchy. This requirement significantly narrows down the possible configurations.

There are two options regarding the $S U(5)$ gauge group. First, it could be related to a single node, namely it is a subgroup of a single $U(N)$ symmetry. In this scenario, the fields transforming as $\mathbf{1 0}$ must have both ends on the $S U(5)$-related node. Consequently they all carry the same $U(1)_{\mathrm{FN}}$ charge, regardless of whether $U(1)_{\mathrm{FN}}$ is (i) a subgroup of the same $U(N)$, (ii) unrelated to this $U(N)$ or (iii) a subgroup of $U(N) \times U(M)$, where $U(M)$ is related to a different node. Thus, this scenario gives rise to up mass anarchy (that is, no special structure in the up mass matrix) and is therefore phenomenologically excluded.

The second scenario has the $S U(5)$ gauge group related to two nodes, namely it is a subgroup of a $U\left(N_{L}\right) \times U\left(N_{R}\right)$ symmetry. The FN field must be in the bifundamental $\left(\mathbf{N}_{\mathbf{L}}, \overline{\mathbf{N}}_{\mathbf{R}}\right)$ of the two nodes. This case has a rich flavor structure and is the only one that can lead to phenomenologically viable models.

The simplest models have the following pattern of gauge symmetry breaking: $S U(5) \times S U(5) \rightarrow S U(5)_{\text {diag }}$. We focus on this class of models. (More complicated breaking patterns have a similar hierarchical form, but involve extended particle content.) The $\overline{\mathbf{5}}$-plets then transform under the $S U(5) \times S U(5) \times U(1)_{\mathrm{L}} \times U(1)_{\mathrm{R}}$ as either $(\overline{\mathbf{5}}, 1)_{-1,0}$ or $(1, \overline{\mathbf{5}})_{0,-1}$. The $\mathbf{1 0}$-plets transform as either $(\mathbf{1 0}, 1)_{+2,0}$ or $(1, \mathbf{1 0})_{0,+2}$ or $(\mathbf{5}, \mathbf{5})_{+1,+1}$. The $H_{u}(\mathbf{5})$ field transforms as either $(\mathbf{5}, 1)_{+1,0}$ or $(1, \mathbf{5})_{0,+1}$.

While $S$ breaks the $U(1)_{\mathrm{FN}}=U(1)_{\mathrm{L}-\mathrm{R}}$ symmetry, it leaves $U(1)_{\mathrm{L}+\mathrm{R}}$ as a global symmetry in the $S U(5)_{\text {diag }}$ theory, under which the fields are charged as

$$
\overline{\mathbf{5}}(-1), \mathbf{1 0}(+2), H_{d}(-1), H_{u}(+1) .
$$

This symmetry is flavor diagonal, with charges that are determined solely through the $S U(5)$ representation. As mentioned in section [C] such $U(1)$ 's are generic and face strong phenomenological constraints. Clearly, the symmetry of eq. (9) is not a symmetry of the $S U(5)$ GUT model. In particular, no up-type masses are allowed with the above symmetry $[\underline{8},[9]$. One can try to overcome this problem by introducing a composite $H_{u}$ field of charge -4 [8]. This solution to the up mass(lessness) problem comes, however, at the cost of two new problems: First, the $H_{u} H_{d}$ term is now forbidden, rendering the higgsinos massless. Second, the $\overline{\mathbf{5}} \overline{\mathbf{5}} H_{u} H_{u}$ terms are forbidden, rendering the neutrinos massless.

As discussed in section $11 \mathrm{C}$ there are three possible solutions to this problem, which involve either extending the particle content and the symmetry of the low energy theory, or breaking the symmetry either spontaneously or non-perturbatively. Before going into details, we observe that, in fact, the quiver theory gives interesting predictions that are independent of which solution to the up mass problem is employed.

Indeed, since the $\overline{\mathbf{5}}$ fields carry $U(1)_{\mathrm{L}} \times U(1)_{\mathrm{R}}$ charges of either $(-1,0)$ or $(0,-1)$, at least two of them have the same FN charge. Thus, there must be at least quasianarchy (that is two non-hierarchical masses and one mixing angle of order one) in the neutrino sector. In half of the models all three $\overline{\mathbf{5}}$ fields have the same FN charge, leading to complete neutrino mass anarchy (no hierarchy in the masses and all three angles of order one). In addition, this situation, where a maximum of two possible FN charges are available to the three $\overline{\mathbf{5}}$ fields, has implications for the down sector: either one or all three (in correspondence to quasi- or full-anarchy in the neutrinos) down mass ratios are of the same order as the corresponding mixing angles (e.g. $\left.m_{s} / m_{b} \sim\left|V_{c b}\right|\right)$.

A word of caution is, however, in order. The above restrictions on the possible FN charges can be evaded if the $\mathbf{1 0}$ and $\overline{\mathbf{5}}$-plets are composite fields 52 . In such a case, it is possible for all fields to carry various charges under additional $U(1)$ factors, which may play the role of the FN symmetry. Such a possibility, however, complicates the theory considerably and does not seem to be attractive, especially since the effective theory cannot be described by a quiver. We thus assume that the SM fermions are elementary fields, while condensates can either break the $U(1)_{\mathrm{L}+\mathrm{R}}$ symmetry or generate effective Higgs fields.

We next discuss the possibilities for solving the $U(1)_{\mathrm{L}+\mathrm{R}}$ problem. We do so in the specific context of $S U(5) \mathrm{GUT}$, but the solutions can be straightforwardly generalized to other gauge groups. 


\section{B. Extending the Higgs Sector}

To allow for up-quark, higgsino and neutrino masses in a model that has the $U(1)_{\mathrm{L}+\mathrm{R}}$ symmetry, one needs to add matter fields. The simplest extension has a second pair of Higgs doublets [5]. The $U(1)_{\mathrm{L}+\mathrm{R}}$ charges of the four Higgs fields are as follows:

$$
H_{u}(-4), H_{d}(-1), \quad h_{d}(+4), h_{u}(+1) .
$$

Here $H_{d}$ and $h_{u}$ are fundamental fields, while $H_{u}$ and $h_{d}$ are composite.

One can now distinguish between models according to the FN charges of the matter fields, that is the three $\mathbf{1 0}_{i}$, the three $\overline{\mathbf{5}}_{i}$, the two elementary Higgs fields $H_{d}$ and $h_{u}$, and the two composite Higgs fields $H_{u}$ and $h_{d}$. There are 640 different sets of $U(1)_{\mathrm{FN}}=U(1)_{\mathrm{L}-\mathrm{R}}$ charges. They are listed in table IV

We now impose phenomenological requirements to see, first, if there are viable models and, second, if these models make further predictions. It turns out that requiring that the quark masses are hierarchical is enough to select a single flavor structure for all fermions and, in particular, predict the flavor structure of the lepton sector.

We first consider the up sector. We require that the three up-type quarks have masses and that these masses are hierarchical. This means that no two 10-plets are allowed to carry the same FN charge. Out of the ten different sets of charges for the 10-plets $T_{i}$, only one fulfills this requirement, that is $T_{5}$ of table IV] Furthermore, the up sector couples to the composite $H_{u}$. In order that the up masses do not vanish, $H_{u}$ must carry charge $(-4,0)$ under $U(1)_{\mathrm{L}} \times U(1)_{\mathrm{R}}$. Thus, of the four sets $U_{i}$, only $U_{1}$ and $U_{2}$ are viable charge assignments.

We next consider the down sector. We require that the three down-type quarks have masses and that these masses are hierarchical. Out of the four different sets of charges for the $\overline{\mathbf{5}}$-plets $F_{i}$, only one fulfills this requirement, that is $F_{1}$. Furthermore, the down sector couples to the elementary $H_{d}$. $H_{d}$ must be connected to the same node as the $\overline{\mathbf{5}}$. Thus, of the four sets $D_{i}$, only $D_{1}$ and $D_{2}$ are viable choices.

We are therefore left with a unique set of $U(1)_{\mathrm{L}-\mathrm{R}}$ charges, up to the choice of the charges for $h_{d}$ and $h_{u}$. This freedom, however, only affects the $\mu$-terms and the overall scale of the neutrino masses. The flavor structure is unaffected by this choice. Taking the configuration " $T_{5} F_{1} D_{1} U_{1}$," we obtain the following parametric suppressions for the various entries in the fermion mass matrices:

$$
\begin{aligned}
& M_{u} \sim\left\langle H_{u}\right\rangle\left(\begin{array}{ccc}
\epsilon^{4} & \epsilon^{3} & \epsilon^{2} \\
\epsilon^{3} & \epsilon^{2} & \epsilon \\
\epsilon^{2} & \epsilon & 1
\end{array}\right), \\
& M_{d} \sim\left\langle H_{d}\right\rangle\left(\begin{array}{ccc}
\epsilon^{2} & \epsilon^{2} & \epsilon^{2} \\
\epsilon & \epsilon & \epsilon \\
1 & 1 & 1
\end{array}\right), \\
& M_{\nu} \sim \frac{\left\langle h_{u}\right\rangle^{2}}{M}\left(\begin{array}{lll}
1 & 1 & 1 \\
1 & 1 & 1 \\
1 & 1 & 1
\end{array}\right) .
\end{aligned}
$$

There are other ways to extend the matter content in order to incorporate the $U(1)_{L+R}$ as a the symmetry of the theory. One way to generate the $\mu$-term for the $H_{u} H_{d}$ fields, without introducing another pair of higgs fields, can be achieved in a similar fashion to the NMSSM. One assumes $H_{u}$ is a condensate $\overline{\mathbf{5}} \overline{\mathbf{5}} \overline{\mathbf{5}}$ and introduces another field $T$ which is a $\mathbf{5 5 5 5 5}$ condensate of the same strongly coupled gauge group. Then the coupling $T H_{u} H_{d}$ is allowed, while the coupling $T^{3}$ which breaks the $U(1)_{\mathrm{L}+\mathrm{R}}$, may be generated by non-perturbative effects below the scale at which the $S U(5)_{\text {diag }}$ becomes strong, or may not be generated at all, as in the MNSSM [45, 46, 47]. We do not discuss this idea further, and just note that, as before, the neutrino sector is predicted to be anarchical.

\section{Breaking $U(1)_{\mathrm{L}+\mathrm{R}} \rightarrow \mathbb{Z}_{5}$}

Another way to get the up Yukawa terms is by breaking the symmetry through non-perturbative effects. In general, operators which violate an anomalous symmetry are generated non-perturbatively and can be calculated using the holomorphic structure of the superpotential. It is important to note that the Lagrangian of the minimal $S U(5)$ posseses a $\mathbb{Z}_{5}$ symmetry under which the fields are charged as in eq. (9). This $\mathbb{Z}_{5}$ is a subgroup of $U(1)_{\mathrm{L}+\mathrm{R}}$. In our case, of an $S U(5)_{\mathrm{L}} \times S U(5)_{\mathrm{R}}$ gauge group, it is simple to see that if in one of the nodes, say the left, there is only a single antisymmetric $\mathbf{1 0}$ (and another $\overline{\mathbf{5}}$ to cancel gauge anomalies), then the related $\Lambda_{\mathrm{QCD}}^{b}$, where $b$ is the coefficient in the $\beta$ function, is charged 5 under the anomalous symmetry. Therefore, instantons, if exist, break this $U(1)$ down to $\mathbb{Z}_{5}$ and can generate masses in the up sector. Unfortunately, at the field theory level, no non-perturbative terms are generated in the superpotential even in this case. The reason is that the number of flavors in this model is $\geq 5$ [48, 49].

Nevertheless, it could be that non-perturbative corrections which break the anomalous $U(1)$ arise already at the string level. Such corrections cannot be calculated explicitly. However, they break the $U(1)$ in a way that follows from the anomaly and hence may generate the required up quark Yukawa couplings. Assuming that these terms are indeed generated in this way, we consider the set of models where there is a single antisymmetric repre- 
sentation on one of the nodes. The list of the 80 possible charge assignments is given in table $\nabla$

Just as for the previous case, most of the possible charge assignments lead to a phenomenologically excluded models. We find again that up mass hierarchy requires that the three 10-plets have three different charges $\left(T_{5}\right)$, and down mass hierarchy requires that the $\overline{\mathbf{5}}$ and $H_{d}$ fields connect to the left node $\left(F_{1} D_{1}\right)$ in which the $U(1)$ is broken down to $\mathbb{Z}_{5}$. Both possible charge assignments for $H_{u}$ give non-vanishing hierarchical masses. Note, however, that $U_{2}$ gives an overall suppression of order $\epsilon$ in the up sector. The neutrino flavor structure is again anarchical (independent of the choice $U_{i}$, though the overall scale depends once again on this choice).

The last class of models involves spontaneous breaking of $U(1)_{L+R}$. This can be achieved by adding a condensate of five fields in the fundamental representations of one $S U(5)$ factor. This condensate includes a singlet of the non-Abelian gauge group with a $U(1)_{\mathrm{L}+\mathrm{R}}$ charge +5 , which we denote by $K$. By giving $K$ a VEV, we break $U(1)_{\mathrm{L}+\mathrm{R}} \rightarrow \mathbb{Z}_{5}$ and allow up type mass terms. A second, conjugate, field $\bar{K}$ is needed in order to allow for a mass term. The list of the 320 different charge sets is given in table VI The majority of the sets of charges are not viable. Only two models are viable: $T_{5} F_{1} D_{1} U_{1} K_{1}$ and $T_{5} F_{1} D_{1} U_{2} K_{1}$, where the latter, as in the previous case, has an overall suppression in the up sector. Once again, anarchy is predicted for the neutrino sector.

In all three classes of models, we arrived at an essentially equivalent configuration for the matter content. The differences between the three models are just with respect to new fields that are added to solve the $U(1)_{\mathrm{L}+\mathrm{R}}$ problem. This unique configuration, which leads to the flavor structure of eq. (11), is presented in the quiver of Fig. 9. (The theory described by this quiver diagram suffers from non-Abelian gauge anomalies. A nonanomalous extension is presented in appendix A and Fig. 10]) This theory produces, at low energy, the minimal $S U(5)$ with the correct hierarchy in the up and down sector and with the predicted neutrino anarchy. We stress that, given our assumptions, this theory is unique and thus the anarchy is predicted.

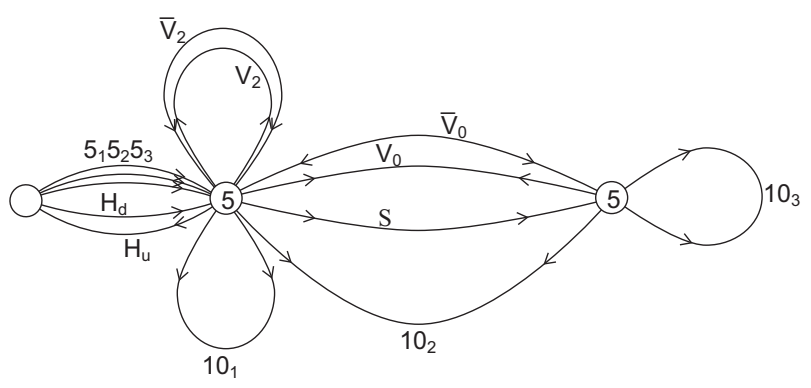

FIG. 9: The unique configuration for the SU(5) GUT fields.

\section{SUMMARY}

The fermion flavor parameters of the Standard Model have a special, non-generic structure, that finds no explanation within the Standard Model. The hierarchy and the smallness of the Yukawa couplings are suggestive of an approximate symmetry. The Froggatt-Nielsen (FN) mechanism is a simple and attractive realization of this idea. The mechanism is, however, limited in its predictive power. In particular, the FN charges are not dictated by the theory, and there is only information on the parametric suppression, but not the order one coefficients, of the Yukawa couplings. In this work, we investigated whether the embedding of the FN mechanism in string theory improves its predictive power.

Specifically, we examined quiver gauge theories which arise at low energy from type II string theory with Dbranes placed on singular manifolds. The quiver gauge theories can be described by (non-)directed graphs for (un)oriented strings, in which the nodes represent the gauge groups $(U(N), S O(N)$ or $S p(N))$, while lines represent matter fields charged under the corresponding gauge groups.

In general, these theories contain several anomalous $U(1)$ symmetries whose anomaly is canceled by a generalized GS mechanism. The unbroken global symmetries can be used to generate the hierarchy of the Yukawa couplings, thus realizing the FN mechanism. Since charges of the matter fields under these $U(1)$ factors are fixed by their representation under the gauge groups, the FN charges are fixed. Consequently, one of the inherent limitations of FN models is removed.

Preciesly because it is highly predictive, the above framework does not easily lend itself to the construction of viable models. We have discussed this problem and its possible solutions. Concentrating on a large class of $S U(5)$ GUT models, we have demonstrated the predictive power of quiver gauge theories. Requiring mass hierarchy in the up sector, we showed that the $S U(5)$ must come from an extended product group such as $U(5) \times U(5)$. Furthermore, there must be either quasior full-anarchy in the neutrino sector and either one or all three down mass ratios are of the same order as the corresponding mixing angles (e.g. $\left.m_{s} / m_{b} \sim\left|V_{c b}\right|\right)$. Further requiring mass hierarchy in the down sector, the FN charges of all matter fields are essentially fixed. Consequently, the lepton flavor structure is predicted and, in particular, there is anarchy (that is, no special structure) in the neutrino sector.

\section{ACKNOWLEDGEMENT}

We are grateful to Micha Berkooz and Yael Shadmi for collaboration in early stages of this work. We thank Ofer Aharony, Tom Banks, Michael Dine, Guy Engelhard, Amihay Hanany, Eric Poppitz, Guy Raz, Martin Schmaltz and Adam Schwimmer for useful discussions. 
The work of YN and TV is supported by a grant from the United States-Israel Binational Science Foundation (BSF), Jerusalem, Israel. YN is supported by the Israel Science Foundation founded by the Israel Academy of Sciences and Humanities, by G.I.F., the German-Israeli Foundation for Scientific Research and Development, by EEC RTN contract HPRN-CT-00292-2002, and by the Minerva Foundation (München).

\section{APPENDIX A: AN SU(5) GUT QUIVER}

As we showed in section IV all three classes of models have an equivalent configuration for the matter content, which leads to the flavor structure of eq. (11). A non-anomalous quiver which realizes this structure is presented in Fig. 10] This figure corresponds specifically to the method of spontaneously breaking the $U(1)_{\mathrm{L}+\mathrm{R}}$ symmetry. In particular, we explicitly show the condensate field, $K$.

Note that the two $S U(5)$ gauge groups are not asymptotically free, which is a typical problem in realizations of the FN mechanism [2]. In our framework, however, the absence of asymptotic freedom does not pose a problem since the theory is defined at the string scale, where new heavy degrees of freedom are integrated in. The two $S U(5)$ factors are broken into the diagonal $S U(5)$ after giving a VEV to the Froggatt-Nielsen field $S$. The fields $\mathbf{1 0}_{\mathbf{i}}, \overline{\mathbf{5}}_{\mathbf{i}}, H_{u}$ and $H_{d}$, together with $\bar{S}$ that becomes an adjoint, are the matter content of $S U(5)$ GUT. The fields denoted by $V_{i}$ are the vector-like fields discussed in section III

All other fields are necessary for non-Abelian gauge anomaly cancellation. The three $X$ fields are connected to a $U(1)$ gauge group. However, to cancel the anomalies, we can alternatively employ a single field connected to a $U(3)$ group. All these additional fields have mass terms in the superpotential and can be integrated out in pairs: $X_{i}$ with $\bar{X}_{i}$ and $\bar{A}_{15}$ with the symmetric part of $\mathbf{1 0}_{2}$. At the level of the massless spectrum, these fields may obtain masses through couplings to $S$ or to other moduli which obtain VEVs and therefore, by definition, are not shown in our effective quiver.

Reverse geometrically engineering a singular string theory background with this quiver is a complicated task. A generic construction is known for a very limited number of cases [38, 50, 51]. In fact, we view our model as an effective quiver obtained by Higging a larger quiver. One reason for this is that the (symmetric) $\bar{A}_{15}$ field cannot be directly obtained together with the (antisymmetric) $\overline{\mathbf{1 0}}$ field through the orientifold projection. Nevertheless, the symmetric $\bar{A}_{15}$ can originate from a broken $S U(5) \times S U(5) \rightarrow S U(5)$ if a $\overline{\mathbf{5}} \times \overline{\mathbf{5}}$ field is broken down to a $\bar{A}_{15}$ and a $\overline{\mathbf{1 0}}$, where the latter is integrated out with another $\mathbf{1 0 .}$

Thus indeed this theory produces, at low energy, the minimal $S U(5)$ with the correct hierarchy in the up and down sector and with the predicted neutrino anarchy.
[1] C. D. Froggatt and H. B. Nielsen, Nucl. Phys. B 147, 277 (1979).

[2] M. Leurer, Y. Nir and N. Seiberg, Nucl. Phys. B 398, 319 (1993) arXiv:hep-ph/9212278; Nucl. Phys. B 420, 468 (1994) arXiv:hep-ph/9310320.

[3] Y. Grossman and Y. Nir, Nucl. Phys. B 448, 30 (1995) arXiv:hep-ph/9502418.

[4] Y. Nir and G. Raz, Phys. Rev. D 66, 035007 (2002) arXiv:hep-ph/0206064.

[5] L. E. Ibanez, F. Marchesano and R. Rabadan, JHEP 0111, 002 (2001) arXiv:hep-th/0105155.

[6] C. Kokorelis, JHEP 0209, $029 \quad$ (2002) arXiv:hep-th/0205147; JHEP 0208, 036 (2002) arXiv:hep-th/0206108.

[7] J. F. G. Cascales, M. P. Garcia del Moral, F. Quevedo and A. M. Uranga, JHEP 0402, 031 (2004) arXiv:hep-th/0312051.

[8] R. Blumenhagen, B. Kors, D. Lust and T. Ott, Nucl. Phys. B 616, 3 (2001) arXiv:hep-th/0107138.

[9] R. Blumenhagen, M. Cvetic, P. Langacker and G. Shiu, arXiv:hep-th/0502005

[10] V. Braun, Y. H. He, B. A. Ovrut and T. Pantev, Phys. Lett. B 618, 252 (2005) arXiv:hep-th/0501070; JHEP 0506, 039 (2005) arXiv:hep-th/0502155; arXiv:hep-th/0512177

[11] V. Braun, Y. H. He and B. A. Ovrut, arXiv:hep-th/0602073

[12] V. Bouchard and R. Donagi, Phys. Lett. B 633, 783

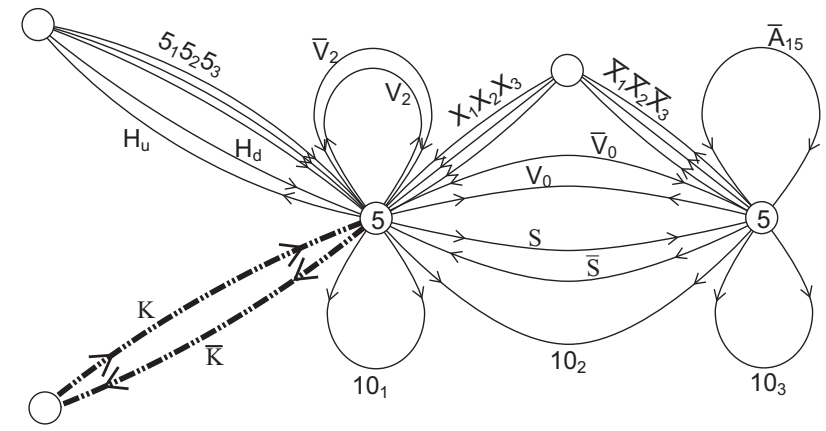

FIG. 10: An SU(5) GUT Quiver.

(2006) arXiv:hep-th/0512149.

[13] V. Bouchard, M. Cvetic and R. Donagi, arXiv:hep-th/0602096

[14] J. Giedt, G. L. Kane, P. Langacker and B. D. Nelson, Phys. Rev. D 71, 115013 (2005) arXiv:hep-th/0502032.

[15] L. E. Ibanez and G. G. Ross, Phys. Lett. B 332, 100 (1994) arXiv:hep-ph/9403338.

[16] V. Jain and R. Shrock, Phys. Lett. B 352, 83 (1995) arXiv:hep-ph/9412367.

[17] P. Binetruy and P. Ramond, Phys. Lett. B 350, 49 (1995) arXiv:hep-ph/9412385.

[18] E. Dudas, S. Pokorski and C. A. Savoy, Phys. Lett. B 
356, 45 (1995) arXiv:hep-ph/9504292.

[19] Y. Nir, Phys. Lett. B 354, 107 (1995) arXiv:hep-ph/9504312.

[20] J. K. Elwood, N. Irges and P. Ramond, Phys. Lett. B 413, 322 (1997) arXiv:hep-ph/9705270; Phys. Rev. Lett. 81, 5064 (1998) arXiv:hep-ph/9807228.

[21] P. Binetruy, S. Lavignac and P. Ramond, Nucl. Phys. B 477, 353 (1996) arXiv:hep-ph/9601243.

[22] H. K. Dreiner, H. Murayama and M. Thormeier, Nucl. Phys. B 729, 278 (2005) arXiv:hep-ph/0312012.

[23] R. Harnik, D. T. Larson, H. Murayama and M. Thormeier, Nucl. Phys. B 706, 372 (2005) arXiv:hep-ph/0404260.

[24] For a different approach to hierarchy in D-brane constructions, see D. Cremades, L. E. Ibanez and F. Marchesano, JHEP 0307, 038 (2003) arXiv:hep-th/0302105 and references therein.

[25] M. R. Douglas and G. W. Moore, arXiv:hep-th/9603167

[26] C. V. Johnson and R. C. Myers, Phys. Rev. D 55, 6382 (1997) arXiv:hep-th/9610140.

[27] A. E. Lawrence, N. Nekrasov and C. Vafa, Nucl. Phys. B 533, 199 (1998) arXiv:hep-th/9803015.

[28] E. Kiritsis, Fortsch. Phys. 52, $200 \quad$ (2004) arXiv:hep-th/0310001.

[29] G. Aldazabal, L. E. Ibanez, F. Quevedo and A. M. Uranga, JHEP 0008, 002 (2000) arXiv:hep-th/0005067.

[30] Y. H. He, arXiv:hep-th/0408142

[31] L. E. Ibanez, R. Rabadan and A. M. Uranga, Nucl. Phys. B 542, 112 (1999) arXiv:hep-th/9808139. Nucl. Phys. B 576, 285 (2000) arXiv:hep-th/9905098.

[32] M. B. Green and J. H. Schwarz, Phys. Lett. B 149, 117 (1984).

[33] M. Dine, N. Seiberg and E. Witten, Nucl. Phys. B 289, 589 (1987).

[34] L. J. Dixon, J. A. Harvey, C. Vafa and E. Witten, Nucl. Phys. B 261, 678 (1985); Nucl. Phys. B 274, 285 (1986).
[35] S. Kachru and E. Silverstein, Phys. Rev. Lett. 80, 4855 (1998) arXiv:hep-th/9802183.

[36] M. Berkooz and R. G. Leigh, Nucl. Phys. B 483, 187 (1997) arXiv:hep-th/9605049.

[37] S. Benvenuti, S. Franco, A. Hanany, D. Martelli and J. Sparks, JHEP 0506, 064 (2005) arXiv:hep-th/0411264.

[38] B. Feng, A. Hanany and Y. H. He, Nucl. Phys. B 595, 165 (2001) arXiv:hep-th/0003085.

[39] I. R. Klebanov and E. Witten, Nucl. Phys. B 536, 199 (1998) arXiv:hep-th/9807080.

[40] D. Martelli and J. Sparks, arXiv:hep-th/0411238

[41] M. Cvetic, G. Shiu and A. M. Uranga, Nucl. Phys. B 615, 3 (2001) arXiv:hep-th/0107166

[42] Z. Lalak, S. Lavignac and H. P. Nilles, Nucl. Phys. B 559, 48 (1999) arXiv:hep-th/9903160.

[43] J. D. Lykken, E. Poppitz and S. P. Trivedi, Nucl. Phys. B 543, 105 (1999) arXiv:hep-th/9806080.

[44] E. Witten, Phys. Lett. B 149, 351 (1984).

[45] C. Panagiotakopoulos and K. Tamvakis, Phys. Lett. B 469, 145 (1999) arXiv:hep-ph/9908351.

[46] C. Panagiotakopoulos and A. Pilaftsis, Phys. Rev. D 63, 055003 (2001) arXiv:hep-ph/0008268.

[47] A. Dedes, C. Hugonie, S. Moretti and K. Tamvakis, Phys. Rev. D 63, 055009 (2001) arXiv:hep-ph/0009125.

[48] E. Poppitz and S. P. Trivedi, Phys. Lett. B 365, 125 (1996) arXiv:hep-th/9507169.

[49] P. Pouliot, Phys. Lett. B 367, 151 (1996) arXiv:hep-th/9510148.

[50] D. Berenstein, JHEP 0204, $052 \quad$ (2002) arXiv:hep-th/0201093.

[51] B. Feng, A. Hanany and Y. H. He, JHEP 0108, 040 (2001) arXiv:hep-th/0104259.

[52] We thank Micha Berkooz for drawing our attention to this point. 


\begin{tabular}{l|c} 
Charges & Suppression \\
\hline \hline$(-1,+1)(-1,+1)(-1,+1)$ & 3 \\
$(0,0)(-1,+1)(-1,+1)$ & 2 \\
$(-1,0)(0,+1)(-1,+1)$ & 2 \\
$(0,0)(0,0)(-1,+1)$ & 1 \\
$(0,0)(-1,0)(0,+1)$ & 1 \\
$(0,+1)(0,-1)(-1,+1)$ & 1 \\
$(+1,0)(-1,0)(-1,+1)$ & 1 \\
$(+1,-1)(-1,+1)(-1,+1)$ & 1 \\
\hline
\end{tabular}

TABLE I: Possible $U(1)_{\mathrm{L}} \times U(1)_{\mathrm{R}}$ charges and the resulting suppression factors (namely the power in $\epsilon$ ) in oriented strings.

\begin{tabular}{l|c} 
Charges & Suppression \\
\hline \hline$(-2,0)(0,+2)(-1,+1)$ & 3 \\
$(-2,0)(0,+2)(0,0)$ & 2 \\
$(-2,0)(+1,+1)(-1,+1)$ & 2 \\
$(-2,0)(0,+1)(0,+1)$ & 2 \\
$(0,+2)(-1,-1)(-1,+1)$ & 2 \\
$(0,+2)(-1,0)(-1,0)$ & 2 \\
$(-2,0)(0,+2)(+1,-1)$ & 1 \\
$(-2,0)(+2,0)(-1,+1)$ & 1 \\
$(-2,0)(+1,+1)(0,0)$ & 1 \\
$(-2,0)(+1,0)(0,+1)$ & 1 \\
$(0,+2)(0,-2)(-1,+1)$ & 1 \\
$(0,+2)(-1,-1)(0,0)$ & 1 \\
$(0,+2)(-1,0)(0,-1)$ & 1 \\
$(+1,+1)(-1,-1)(-1,+1)$ & 1 \\
$(+1,+1)(-1,0)(-1,0)$ & 1 \\
$(-1,-1)(0,+1)(0,+1)$ & 1 \\
\hline
\end{tabular}

TABLE II: Possible charges and suppression factors (the power of $\epsilon$ ) with $S(+1,-1)$ that are specific to the unoriented case. The sets of table \are also allowed. 


\begin{tabular}{l|c} 
Charges & Suppression \\
\hline \hline$(-2,0)(-2,0)(-2,0)$ & 3 \\
$(-2,0)(-2,0)(0,0)$ & 2 \\
$(-2,0)(-1,-1)(-1,1)$ & 2 \\
$(-2,0)(-1,0)(-1,0)$ & 2 \\
$(-2,0)(-2,0)(+2,0)$ & 1 \\
$(-2,0)(0,-2)(0,+2)$ & 1 \\
$(-2,0)(-1,-1)(+1,+1)$ & 1 \\
$(-2,0)(0,0)(0,0)$ & 1 \\
$(-2,0)(-1,0)(+1,0)$ & 1 \\
$(-2,0)(0,-1)(0,+1)$ & 1 \\
$(-2,0)(-1,+1)(+1,-1)$ & 1 \\
$(0,-2)(-1,+1)(-1,+1)$ & 1 \\
$(0,2)(-1,-1)(-1,-1)$ & 1 \\
$(-1,-1)(0,0)(-1,+1)$ & 1 \\
$(-1,-1)(-1,0)(0,+1)$ & 1 \\
$(0,0)(-1,0)(-1,0)$ & 1 \\
$(-1,0)(0,-1)(-1,+1)$ & 1 \\
\hline
\end{tabular}

TABLE III: Possible charges and suppression factors (the power of $\epsilon$ ) in the unoriented case with $S(+2,0)$. The $S(-2,0)$ case is obtained by multiplying the $U(1)_{\mathrm{L}}$-charge by -1 . 


\begin{tabular}{|c|c|c|c|}
\hline$S U(5)$ & Model & $U(1)_{L} \times U(1)_{R}$ & $U(1)_{L-R}$ \\
\hline \multirow[t]{10}{*}{$10_{i}(i=1,2,3)$} & $T_{1}$ & $(2,0)(2,0)(2,0)$ & $(+2,+2,+2)$ \\
\hline & $T_{2}$ & $(2,0)(2,0)(0,2)$ & $(+2,+2,-2)$ \\
\hline & $T_{3}$ & $(2,0)(2,0)(1,1)$ & $(+2,+2,0)$ \\
\hline & $T_{4}$ & $(2,0)(0,2)(0,2)$ & $(+2,-2,-2)$ \\
\hline & $T_{5}$ & $(2,0)(0,2)(1,1)$ & $(+2,-2,0)$ \\
\hline & $T_{6}$ & $(2,0)(1,1)(1,1)$ & $(+2,0,0)$ \\
\hline & $T_{7}$ & $(0,2)(0,2)(0,2)$ & $(-2,-2,-2)$ \\
\hline & $T_{8}$ & $(0,2)(0,2)(1,1)$ & $(-2,-2,0)$ \\
\hline & $T_{9}$ & $(0,2)(1,1)(1,1)$ & $(-2,0,0)$ \\
\hline & $T_{10}$ & $(1,1)(1,1)(1,1)$ & $(0,0,0)$ \\
\hline \multirow[t]{4}{*}{$\overline{5}_{i}(i=1,2,3)$} & $F_{1}$ & $(-1,0)(-1,0)(-1,0)$ & $(-1,-1,-1)$ \\
\hline & $F_{2}$ & $(-1,0)(-1,0)(0,-1)$ & $(-1,-1,+1)$ \\
\hline & $F_{3}$ & $(-1,0)(0,-1)(0,-1)$ & $(-1,+1,+1)$ \\
\hline & $F_{4}$ & $(0,-1)(0,-1)(0,-1)$ & $(+1,+1,+1)$ \\
\hline \multirow[t]{4}{*}{$H_{d}(\overline{5}), h_{u}(5)$} & $D_{1}$ & $(-1,0),(+1,0)$ & $(-1,+1)$ \\
\hline & $D_{2}$ & $(-1,0),(0,+1)$ & $(-1,-1)$ \\
\hline & $D_{3}$ & $(0,-1),(+1,0)$ & $(+1,+1)$ \\
\hline & $D_{4}$ & $(0,-1),(0,+1)$ & $(+1,-1)$ \\
\hline \multirow[t]{4}{*}{$H_{u}(5), h_{d}(\overline{5})$} & $U_{1}$ & $(-4,0),(+4,0)$ & $(-4,+4)$ \\
\hline & $U_{2}$ & $(-4,0),(0,+4)$ & $(-4,-4)$ \\
\hline & $U_{3}$ & $(0,-4),(+4,0)$ & $(+4,+4)$ \\
\hline & $U_{4}$ & $(0,-4),(0,+4)$ & $(+4,-4)$ \\
\hline$S(1)$ & & $(+1,-1)$ & +2 \\
\hline
\end{tabular}

TABLE IV: All possible charge assignments for the model with additional fields and an unbroken $U(1)_{L+R}$ symmetry 


\begin{tabular}{l|c|c|c}
$S U(5)$ & Model & $U(1)_{L} \times U(1)_{R}$ & $U(1)_{L-R}$ \\
\hline \hline $10_{i}$ & $T_{2}$ & $(2,0)(2,0)(0,2)$ & $(+2,+2,-2)$ \\
& $T_{4}$ & $(2,0)(0,2)(0,2)$ & $(+2,-2,-2)$ \\
& $T_{5}$ & $(2,0)(0,2)(1,1)$ & $(+2,-2,0)$ \\
& $T_{6}$ & $(2,0)(1,1)(1,1)$ & $(+2,0,0)$ \\
& $T_{9}$ & $(0,2)(1,1)(1,1)$ & $(-2,0,0)$ \\
& & & \\
$\overline{5}_{i}$ & $F_{1}$ & $(-1,0)(-1,0)(-1,0)$ & $(-1,-1,-1)$ \\
& $F_{2}$ & $(-1,0)(-1,0)(0,-1)$ & $(-1,-1,+1)$ \\
& $F_{3}$ & $(-1,0)(0,-1)(0,-1)$ & $(-1,+1,+1)$ \\
& $F_{4}$ & $(0,-1)(0,-1)(0,-1)$ & $(+1,+1,+1)$ \\
& & & -1 \\
$H_{d}(\overline{5})$ & $D_{1}$ & $(-1,0)$ & +1 \\
& $D_{2}$ & $(0,-1)$ & +1 \\
$H_{u}(5)$ & & & -1 \\
& $U_{1}$ & $(1,0)$ & +2 \\
\hline
\end{tabular}

TABLE V: All possible charge assignments for the model with instanton breaking of the $U(1)_{L+R}$ 


\begin{tabular}{|c|c|c|c|}
\hline$S U(5)$ & Model & $U(1)_{L} \times U(1)_{R}$ & $U(1)_{L-R}$ \\
\hline \multirow[t]{10}{*}{$10_{i}$} & $T_{1}$ & $(2,0)(2,0)(2,0)$ & $(+2,+2,+2)$ \\
\hline & $T_{2}$ & $(2,0)(2,0)(0,2)$ & $(+2,+2,-2)$ \\
\hline & $T_{3}$ & $(2,0)(2,0)(1,1)$ & $(+2,+2,0)$ \\
\hline & $T_{4}$ & $(2,0)(0,2)(0,2)$ & $(+2,-2,-2)$ \\
\hline & $T_{5}$ & $(2,0)(0,2)(1,1)$ & $(+2,-2,0)$ \\
\hline & $T_{6}$ & $(2,0)(1,1)(1,1)$ & $(+2,0,0)$ \\
\hline & $T_{7}$ & $(0,2)(0,2)(0,2)$ & $(-2,-2,-2)$ \\
\hline & $T_{8}$ & $(0,2)(0,2)(1,1)$ & $(-2,-2,0)$ \\
\hline & $T_{9}$ & $(0,2)(1,1)(1,1)$ & $(-2,0,0)$ \\
\hline & $T_{10}$ & $(1,1)(1,1)(1,1)$ & $(0,0,0)$ \\
\hline \multirow[t]{4}{*}{$\overline{5}_{i}$} & $F_{1}$ & $(-1,0)(-1,0)(-1,0)$ & $(-1,-1,-1)$ \\
\hline & $F_{2}$ & $(-1,0)(-1,0)(0,-1)$ & $(-1,-1,+1)$ \\
\hline & $F_{3}$ & $(-1,0)(0,-1)(0,-1)$ & $(-1,+1,+1)$ \\
\hline & $F_{4}$ & $(0,-1)(0,-1)(0,-1)$ & $(+1,+1,+1)$ \\
\hline \multirow[t]{2}{*}{$H_{d}(\overline{5})$} & $D_{1}$ & $(-1,0)$ & -1 \\
\hline & $D_{2}$ & $(0,-1)$ & +1 \\
\hline \multirow[t]{2}{*}{$H_{u}(5)$} & $U_{1}$ & $(+1,0)$ & +1 \\
\hline & $U_{2}$ & $(0,+1)$ & -1 \\
\hline \multirow[t]{2}{*}{$K(1), \bar{K}(1)$} & $K_{1}$ & $(+5,0),(-5,0)$ & $(+5,-5)$ \\
\hline & $K_{2}$ & $(0,+5),(0,-5)$ & $(-5,+5)$ \\
\hline$S(1)$ & & $(+1,-1)$ & +2 \\
\hline
\end{tabular}

TABLE VI: All possible charge assignments for the model with spontaneous breaking of the $U(1)_{L+R}$ symmetry 